\title{
Lymphknoten mit Metastase gleich markieren
}

Das Markieren von Lymphknoten mit Metastasenbefund mit einem Clip kann helfen, das Ansprechen auf eine neoadjuvante Therapie zu beurteilen. Eine gezielte Entfernung der geclippten Lymphknoten zusammen mit den Sentinel-Lymphknoten in der Axilla (Targeted Axillary Dissection, TAD) könnte aber auch die falsch-negative Rate (FNR) der Sentinel-Lymphknotenbiopsie (SLND) alleine verbessern.

n einer prospektiven Studie wurde bei Patientinnen mit bioptisch gesicherten Lymphknotenmetastasen der entsprechende Lymphknoten mit einem Clip markiert. Nach neoadjuvanter Therapie erfolgte die Axilladissektion und die Untersuchung des geclippten Lymphknotens. Eine TAD umfasste die SLND und die Entfernung des markierten Lymphknotens. Die FNR von TAD und SLND alleine wurde anschließend auf Basis einer kompletten Achselausräumung (ALND) bestimmt.

Von 208 Patientinnen hatten sich 191 einer ALND unterzogen. Ein Residuum ließ sich dabei bei 120 (63\%) feststellen. Der wegen einer Metastase markierte Lymphknoten zeigte bei 115 Patientinnen eine Metastase, das Clipping führte also $\mathrm{zu}$ einer FNR von 4,2\% für den markierten Lymphknoten alleine (95\%-Konfidenzintervall [95\%-KI] 1,4-9,5). Patientinnen, bei denen SLND und ALND durchgeführt worden waren $(\mathrm{n}=118)$, wiesen eine FNR von 10,1 \% auf (95\%-KI 4,2-19,8; 7 falsch-negative Ergebnisse bei den 69 Patientinnen mit Residuum). Die Hinzunahme des geclippten Lymphknotens zur SLND verringerte die FNR deutlich auf $1,4 \%$ (95\%KI 0,03-7,3; $\mathrm{p}=0,03)$.
Der markierte Lymphknoten wäre in $23 \%$ der Fälle (31 von 134 Patienten) mit der SLND alleine nicht entdeckt worden. Darunter waren auch sechs Patientinnen mit negativen Sentinel-Lymphknoten, aber einer Metastase im markierten Lymphknoten. Eine TAD erfolgte vor der ALND bei 85 Patientinnen. Hier lag die FNR bei $2,0 \%$ (1 von 50 ).

Fazit: Die Markierung der Lymphknoten mit bioptisch gesicherter Metastase ermöglicht nach der neoadjuvanten Therapie eine selektive Entfernung und pathologische Untersuchung. Zusätzlich zur SLND kann dies die FNR nach dieser explorativen Studie von gut 10 auf $2 \%$ senken. Damit könnte die TAD die erhebliche Morbidität durch die ALND vermindern helfen.

Friederike Klein

Caudle AS et al. Improved Axillary Evaluation Following Neoadjuvant Therapy for Patients With Node-Positive Breast Cancer Using Selective Evaluation of Clipped Nodes: Implementation of Targeted Axillary Dissection. J Clin Oncol. 2016;34(10):1072-8.

\section{Medikamentöser Erhalt der Ovarfunktion bei Brustkrebs überzeugt nicht}

\section{Jungen Frauen mit Brustkrebs steht zum Fertilitätserhalt bei Chemotherapie die Kryokonservierung von Eierstockgewebe zur Verfügung. Nun wurde untersucht, ob auch die Gabe des GnRH-Analogons Triptorelin die ovarielle Funktion erhalten kann, ohne dass es zu Wechselwirkungen oder Inter- aktionen mit der übrigen Therapie kommt.}

A n der Phase-III-Studie nahmen 281 prämenopausale Frauen mit einem Mammakarzinom des Stadiums I-III (Hormonrezeptor [HR]-positiv und -negativ) im Alter von 24-45 Jahren teil. Randomisiert erhielten sie eine adjuvante oder neoadjuvante Chemotherapie alleine oder ergänzt um Triptorelin. HRpositive Patientinnen erhielten nach Ende der Chemotherapie 5 Jahre lang ein endokrine Therapie. Die Nachuntersuchungen erfolgten jährlich. Primärer Endpunkt war die Inzidenz einer chemotherapiebedingten vorzeitigen $\mathrm{Me}$ nopause, Endpunkte einer Post-hocAnalyse waren Langzeitfunktion des Ovars (mindestens eine Menstruation in den letzten 12 Monaten), Schwanger- schaften und krankheitsfreies Überleben (DFS).

Nach median 7,3 Jahren lag die kumulative Inzidenz eines Wiederauftretens der Menstruation über 5 Jahre nach Triptorelin bei $72,6 \%$, in der Kontrollgruppe bei $64,0 \%$ (Hazard Ratio [HR] 1,28; p = $0,07)$. Altersadjustiert ergab sich ein noch etwas deutlicherer Vorteil für die Triptorelin-Gruppe (HR 1,48; $\mathrm{p}=0,006$ ). Hier kam es zu 8 Schwangerschaften (kumulierte 5-Jahres-Inzidenz 2,1\%), in der Kontrollgruppe zu 3 (kumulierte 5-Jahres-Inzidenz 1,6\%; HR 2,56; $\mathrm{p}=0,14$, altersadjustiert: HR 2,40; $p=0,20)$. Die Rate für das 5-Jahres-DFS unterschied sich nicht (Triptorelin 80,5\%, Kontrollgruppe $83,7 \%$; HR 1,17; $\mathrm{p}=0,52$ ).
Fazit: Prämenopausale Patientinnen mit Mammakarzinom profitieren unabhängig von ihrem HR-Status von der Verabreichung von Triptorelin parallel zu einer Chemotherapie hinsichtlich des Wiedereinsetzens der Menstruation. Statistisch signifikant häufiger kam es dabei aber in der Beobachtungszeit nicht zu Schwangerschaften.

Die Nachbeobachtungsfrist für eine endgültige Bewertung ist allerdings auch noch relativ kurz; zumal Frauen mit HRpositivem Mammakarzinom nach der Chemotherapie noch über 5 Jahre eine endokrine Therapie erhielten, sodass eine Schwangerschaft in dieser Gruppe erst deutlich später überhaupt möglich gewesen wäre, so die Forscher. Friederike Klein

Lambertini M et al. Ovarian Suppression With Triptorelin During Adjuvant Breast Cancer Chemotherapy and Long-term Ovarian Function, Pregnancies, and Disease-Free Survival:

A Randomized Clinical Trial. JAMA. 2015; 314(24):2632-40. 